\title{
Influence of summer daylight saving time on scattered erythemal solar ultraviolet exposures
}

\author{
A.V. Parisi ${ }^{*}{ }^{1}$, J. Turner $^{1}$, D.J. Turnbull ${ }^{1}$, P. Schouten ${ }^{1}$ and N. Downs ${ }^{1}$ \\ ${ }^{1}$ Faculty of Sciences, University of Southern Queensland, Toowoomba, 4350. \\ Australia. Ph: 61 (0)7 46312226, Fax: 61 (0)7 46312721. Email: parisi@usq.edu.au \\ *To whom correspondence should be addressed
}

\section{Abstract}

The research question of whether there are any influences in the scattered or diffuse erythemal UV exposures to a horizontal plane over a five month period due to the change from standard time to daylight saving time, has been investigated by using physical measurements and applying them to both standard time and daylight saving time. The diffuse erythemal UV was considered for fixed lunch break times and fixed morning and afternoon break times. The cases considered were for groups of the population who are predominantly indoors and who spend their break times outdoors in shade. The biggest influence on the diffuse UV exposures of changing to daylight saving time is the timing of the outdoor meal and break times. The change causes a reduction in diffuse erythemal exposure for early or morning breaks and an increase in the diffuse erythemal exposure for late or afternoon breaks. Similarly, for the lunch break times, the changes in exposure are influenced by the timing of the break with respect to solar noon. Indoor workers who take their breaks outside in a shaded area may have a change in their exposure to diffuse UV due to a shift to daylight saving time, however the magnitude of this change and whether it is a positive or negative change in exposure will depend on the timing of the break. The increase in diffuse UV exposure due to the afternoon break may be negated by the decrease in exposure due to the morning break. In this case, the effect on diffuse UV exposures due to changing to daylight saving time will be minimal.

Keywords: UV exposure; Diffuse; Daylight saving time; Erythema. 


\subsection{Introduction}

The solar erythemal UV exposures to population groups on specific days of the year depend on the times of the day and the length of time that different population groups spend outdoors along with the weather and atmospheric conditions at those times [1]. UV radiation incident on the Earth's surface, referred to as global UV is comprised of both a direct and a scattered or diffuse component. As the direct component is incident directly from the sun, it is easy to minimize by simply blocking its path. However, the diffuse UV component is incident from all directions due to atmospheric scattering and can contribute significantly to the overall erythemal UV exposures to different population groups [2]. The proportion of diffuse UV radiation to direct UV radiation changes with solar zenith angle (SZA) and cloudiness $[3,4]$ and this influences the diffuse UV exposures to population groups.

Solar UV exposure is a risk factor in a range of sun-related skin and eye diseases. Eye disorders such as cataracts, age-related macular degeneration, pterygium and photokeratitis have been shown to be sun related [5]. Cataracts are a major public health problem, being the primary cause of blindness in humans [6]. Skin cancer is a major health problem that can be reduced through minimisation of UV exposures. On the beneficial side, the UVB waveband (280 to $320 \mathrm{~nm}$ ) acts as an initiator of the synthesis of vitamin $\mathrm{D}_{3}$ for humans by the photolysis of 7-dehydrocholesterol in the human skin, to pre-vitamin $\mathrm{D}_{3}$. The vitamin $\mathrm{D}$ produced by the skin as a result of the action of sunlight, plays an important role in calcium metabolism and the possible reduction of the risk of other diseases [7]. A position statement approved by the Australian and New Zealand Bone and Mineral society, Osteoporosis Australia, the 
Australasian College of Dermatologists and the Cancer Council Australia recommends that in Australia in summer fair skinned people can achieve adequate vitamin D levels by exposing the face, arms and hands or equivalent area of skin to a few minutes of sunlight on either side of the peak UV periods on most days of the week. In winter, in the southern regions of Australia, maintenance of vitamin D levels may require 2-3 hours of sunlight exposure to the face, arms and hands or equivalent area of skin over a week [8].

Consequently, it is essential to optimise solar UV exposures. A strategy to optimise solar UV exposure includes the wearing of sun protective clothing, a hat, the reduction of solar UV exposures through behavioural modification such as the timing and the length of outdoor exposures and the use of shade. The use of shade is widely promoted as one of the strategies for the reduction of solar UV exposures [9]. In these cases where shade is used, the erythemal exposures to population groups while in a shaded environment are due to the diffuse erythemal UV radiation.

Daylight saving time is the practice of shifting local time forward by one hour to try to coincide the rising of the sun with the rising of the individual. It was first introduced as a means to save on heating costs in cooler latitudes. In the countries and states where this occurs, this is generally in late spring, summer and early autumn for mid-latitudes. Many countries worldwide take part in implementing daylight saving time but it is only effective in countries where day is longer than night. For southern hemispheres, daylight saving time is put into effect from September or October to March or April, while the opposite is true for northern hemispheres. In Australia, three of the eastern states practice daylight saving time (AEDT), whereas Queensland 
remains on standard time (AEST). Research has considered the influence of daylight saving time on different aspects, for example the impact on residential energy consumption and cost [10], the influence on motor vehicle and pedestrian fatalities [11] and the influence of the transition to daylight saving time on sleep duration and sleep efficiency [12].

Daylight saving time changes the time with respect to solar noon that population groups who spend any time outdoors are exposed to UV radiation. The changes in global erythemal UV exposures received during different meal break times has been considered for occupational groups due to turning clocks forward by one hour [13]. The results showed that changing to daylight saving time provides an increase in the UV exposures for workers outdoors during certain meal break times and a decrease in the UV exposures for other meal break times. The net UV exposure is therefore dependent on meal break times, for both outdoor and indoor workers.

The diffuse erythemal UV exposures vary due to solar zenith angle, so exposure to diffuse erythemal UV by the population during outdoor break times may be affected by the changing of clock times. This paper will consider the population groups who are predominantly indoors and who venture outdoors for lunch breaks and break times in the morning or afternoon and who choose to sit in a shaded environment. The paper will investigate the research question of whether there are any influences on the diffuse erythemal UV exposures to a horizontal plane due to the change to daylight saving time or summer time from standard time that occurs in some states of Australia and other parts of the world. 


\subsection{Methods}

\subsection{Diffuse UV Measurements}

The diffuse erythemal UV broadband exposures were recorded daily in Toowoomba, Australia $\left(27.5^{\circ} \mathrm{S}, 151.9^{\circ} \mathrm{E}\right)$ on an unshaded building roof, in five minute intervals from dawn to dusk. A UV Biometer (model 510, Solar Light Co., Philadelphia, USA) that normally measures the global erythemal UV was employed to measure the diffuse erythemal UV. The spectral response of the UV Biometer as provided by the manufacturer approximates the erythemal action spectrum [14]. The rooftop where the meter is located and surrounding rooftops are made up of UV reflective surfaces, which contribute to the overall diffuse UV present in the location.

In order to record the diffuse erythemal UV, the meter was fitted with a shadow band that continuously shades the sensor. The shadow band was aligned in an east-west direction, blocking the direct UV sun as the sun traverses across the sky during the day [15]. The angle of the shadow band above the horizontal plane was manually adjusted as required on approximately a weekly basis. The shadow band was $7.6 \mathrm{~cm}$ wide and $25 \mathrm{~cm}$ to $27 \mathrm{~cm}$ from the meter's sensor. The amount of sky view that the shadow band blocks has been measured at approximately $10 \%$ and a correction has been applied to the data to correct for this [15].

The diffuse erythemal UV exposures are recorded in units of MED (minimum erythemal dose) for each five minute interval. An MED is the amount of biologically effective UV required to produce barely perceptible erythema after an interval of 8-24 hours following exposure [16]. At any five minute point, the value recorded is the diffuse erythemal UV exposure for the previous five minutes ending at the respective five minute point. The UV Biometer sensor was temperature stabilised to $25{ }^{\circ} \mathrm{C}$ and 
was calibrated to a UV spectroradiometer (Bentham Instruments Inc, Reading, UK) over a range of SZA. The spectroradiometer recorded the UV spectrum in $0.5 \mathrm{~nm}$ increments and allowed calculation of the erythemal UV irradiances by weighting the spectrum with the erythemal action spectrum. The spectroradiometer was irradiance calibrated against a $150 \mathrm{~W}$ quartz tungsten halogen lamp with calibration traceable to the National Physical Laboratory, UK standard and wavelength calibrated against the UV spectral lines of a mercury lamp. This calibration process provided a calibration of one MED recorded by the meter being equivalent to $277 \mathrm{Jm}^{-2}$ of erythemally weighted UV.

\subsection{Analysis}

In Australia, the current situation is that daylight savings is used in most states of Australia, except for Queensland and the Northern Territory, for approximately four months of the year. The period of the months at the end of 2003 and at the start of 2004 was considered for this paper and is representative of the weather conditions experienced for this time of year in most years. In this case, the two eastern states of New South Wales and Victoria switched from Standard time (AEST) to daylight saving time (AEDT). This started on 26 October 2003 and finished on 28 March, 2004. In comparison, the third eastern state of Queensland did not switch to AEDT and remained on AEST.

The diffuse erythemal UV exposures of occupational groups who have fixed lunch breaks and fixed morning and afternoon break times will be considered. The cases of these population groups spending their lunch breaks and break times outdoors and in the shade and as a result exposed to diffuse UV radiation will be investigated. The UV 
exposures to population groups while in shade is influenced by the diffuse UV to a horizontal plane, the amount of sky view visible from beneath the shade and the orientation of the anatomical site that is receiving the UV exposures. This paper will explore the diffuse erythemal UV to a horizontal plane as this is one of the major contributing factors.

The diffuse erythemal UV exposures recorded at each five minutes to a horizontal plane were employed to determine the diffuse erythemal UV exposures for fixed period lunch break times and fixed morning and afternoon break times. The lunch break times of 11.00-11.30 AEST, 11.30-12.00 AEST, 12.00-12.30 AEST, 12.3013.00 AEST, 13.00-13.30 AEST and 13.30-14.00 AEST were considered and the diffuse erythemal UV exposures over each period determined for the time zones of AEST $\left(\mathrm{UV}_{\mathrm{AEST}}\right)$ and AEDT $\left(\mathrm{UV}_{\mathrm{AEDT}}\right)$ and the difference $\mathrm{UV}_{\mathrm{AEST}}-\mathrm{UV}_{\mathrm{AEDT}}$ calculated. For the morning break times, the diffuse erythemal UV exposures on a horizontal plane for the times of 10.00-10.15 AEST, 10.15-10.30 AEST, 10.30-10.45 AEST and 10.45-11.00 AEST were considered. Similarly, the times of the afternoon break times considered were 14.30-14.45 AEST, 14.45-15.00 AEST, 15.00-15.15 AEST and 15.15-15.30 AEST. The aim was to determine the diffuse erythemal UV exposures to a horizontal plane during these times and no account was made for the orientation or the inclination of the receiving plane under the shade.

In order to calculate the cumulative differences in diffuse exposures, namely $\mathrm{UV}_{\mathrm{AEST}}$ $\mathrm{UV}_{\mathrm{AEDT}}$ over the five months of the daylight saving period, the cumulative differences in exposure for each of the lunch breaks and break times were summed as follows:

$$
U V_{\text {ery }}=\sum_{m} N(m) \sum_{h=0}^{23} \sum_{i=1}^{12} A E(i, h)
$$


where $\mathrm{AE}$ is the difference $\mathrm{UV}_{\mathrm{AEST}}-\mathrm{UV}_{\mathrm{AEDT}}$ in the ambient erythemal diffuse UV exposures on a horizontal plane, i represents each of the five minute intervals in the $\mathrm{h}^{\text {th }}$ hour, $\mathrm{h}$ is the hour of the day, $\mathrm{N}(\mathrm{m})$ is the number of days in the month, $\mathrm{m}$. This is based on a similar technique previously employed to sum the UV exposures over periods of time [17].

\subsection{Results}

The diffuse erythemal UV exposures for each five minute period on the cloud free (less than 10\% cloud) day of 1 February 2004 are plotted in Figure 1 for the AEST times and then shifted one hour for the AEDT times. The exposures are in units of MED for each of the five minute periods. On this day, the minimum solar zenith angle (SZA) was $10.2^{\circ}$ and the SZA at 10.00 AEST was $30.7^{\circ}$. The peak in the diffuse erythemal UV exposures on this day for the AEST time zone was at approximately noon with an exposure of 0.18 MED for each five minutes. This peak exposure occurs for a number of five minute periods over approximately one hour. At this time of the day, the approximate time required for an exposure of $1 \mathrm{MED}$ due to diffuse UV is approximately 28 minutes. At the times of 10:00 AEST and 14:00 AEST, the diffuse UV exposures are 0.14 MED and 0.15 MED respectively for each five minutes. On this day, the times of day at which the diffuse erythemal UV is half of that at the maximum are 8.30 AEST and 15.40 AEST.

For the cases of cloud free days, the diffuse erythemal UV exposures over the five minute periods in the morning are lower for given clock times on AEDT compared to AEST. For example, at 10.00 AEST on this day, the diffuse erythemal UV exposure over five minutes drops from 0.143 MED to 0.108 MED at 10.00 AEDT. This is a difference of 0.035 MED. The opposite occurs in the afternoon where the diffuse 
erythemal UV exposures are higher for given clock times on AEDT compared to AEST. For example, on this day, the diffuse erythemal UV exposures rise from 0.150 MED at 14.00 AEST to 0.172 MED at 14.00 AEDT, an increase of 0.022 MED.

Figure 2 illustrates the changes in diffuse erythemal UV in shifting from AEST to AEDT, on a cloudy day with scattered cloud. The maximum diffuse erythemal UV is 0.290 MED at 12.15 AEST. In this case, the effect on the diffuse erythemal UV exposures of moving from AEST to AEDT is unpredictable as the exposures at a particular time may be higher or lower in both the morning and afternoon, depending on the cloud conditions at the time. This is due to the variability of the influence of clouds on the diffuse UV exposures. On this day, there are cases where the diffuse UV exposures over the five minutes vary by a factor of approximately two over a 20 minute interval. For example at 10.20 AEST, the diffuse erythemal UV is 0.113 MED and at 10.40 AEST, the exposure is 0.240 MED. In this case, the shift from AEST to AEDT time zones results in a drop in the diffuse UV exposure from the value of 0.240 MED at 10.40 AEST to 0.114 MED at 10.40 AEDT.

The differences between the diffuse erythemal UV exposures for the AEST times and the AEDT times received over the fixed lunch break times for each day over the daylight saving period are shown in Figure 3. These are for the lunch break times of 11.00-11.30 AEST, 11.30-12.00 AEST, 12.00-12.30 AEST, 12.30-13.00 AEST, 13.00-13.30 AEST and 13.30-14.00 AEST. In these graphs, day 1 represents the start of the period on 26 Oct 2003 and the days are numbered consecutively from that day. The majority of the differences are between approximately -0.5 and 0.5 MED. However there are some days where the differences are below -0.5 MED and higher 
than 0.5 MED. The averages over all of the days in the five months are $0.50 \pm 0.24$, $0.06 \pm 0.25,-0.005 \pm 0.18,-0.05 \pm 0.16,-0.09 \pm 0.11$ and $-0.17 \pm 0.15$ MED for each of the lunch break times respectively where the error is represented as one standard deviation.

For four different morning and afternoon break times the differences of the diffuse erythemal UV exposures for the AEST times and the AEDT times have been averaged for each month and are shown in Figure 4. The data for the groups 1 to 4 are for the 15 minute morning break times of 10.00-10.15 AEST, 10.15-10.30 AEST, 10.30-10.45 AEST and 10.45-11.00 AEST respectively. The data for the groups 5 to 8 are for the 15 minute afternoon break times of 14.30-14.45 AEST, 14.45-15.00 AEST, 15.00-15.15 AEST and 15.15-15.30 AEST respectively. The averages for each month are variable due to changing cloud cover. However, for the morning meal break times, the differences are positive and for the afternoon meal break times, the differences are negative and the differences range between -0.15 MED and 0.15 MED.

The cumulative diffuse $\mathrm{UV}_{\mathrm{AEST}}-\mathrm{UV}_{\mathrm{AEDT}}$ exposures over the five month period for each of the fixed lunch break times are shown in Figure 5. The largest difference is for either of the early or late lunch break times with practically no difference for the 12.00-12.30 AEST lunch break time. The cumulative diffuse $\mathrm{UV}_{\mathrm{AEST}}-\mathrm{UV}_{\mathrm{AEDT}}$ exposures for each of the fixed morning and afternoon tea break times are in Figure 6. The main influence in this case is whether the break time is in the morning or the afternoon. 


\subsection{Discussion}

The paper has investigated if there are any influences in the diffuse erythemal UV exposures to a horizontal plane over a five month period due to the change from standard time to daylight saving time. The diffuse erythemal UV was considered for fixed lunch break times and fixed morning and afternoon break times. There are possible changes in exposure due to changing the times outdoors with respect to solar noon. The cases considered were for groups of the population who are predominantly indoors and who spend their break times outdoors in shade.

The diffuse UV exposures to a horizontal plane were considered over a five month period consisting of summer and the last month of spring and first month of autumn. The research data took into account the variations in SZA over this time and the variations in cloud cover and atmospheric conditions. The influence of orientation and inclination of the receiving plane and the effect of the amount of sky view in the shade will also influence the diffuse erythemal UV exposures while in shade. For example, reducing the total sky view will contribute to a reduction of diffuse UV radiation. However the aim of this current research was to consider the influence of the change from standard time to daylight saving time on the diffuse erythemal UV available on a horizontal plane and the influences of the receiving plane and the sky view can be considered in future work. The horizontal plane data provides the exposures to parts of the body that have an inclination on a horizontal plane, for example the vertex and possibly parts of the arms or legs if a person is in a sitting position.

The biggest influence on the diffuse UV exposures of changing to AEDT is the timing of the lunch breaks and break times. The change to AEDT causes a reduction in 
diffuse erythemal exposure for early or morning breaks and an increase in the diffuse erythemal exposure for late or afternoon breaks. Similarly, for the lunch break times, the changes in exposure of $\mathrm{UV}_{\mathrm{AEST}}-\mathrm{UV}_{\mathrm{AEDT}}$ are influenced by the timing of the break with respect to solar noon. Other influences on the $U_{\mathrm{AEST}}-\mathrm{UV}_{\mathrm{AEDT}}$ differences in exposure are the effects of cloud, ozone and aerosols. The variations in $\mathrm{UV}_{\mathrm{AEST}}$ $\mathrm{UV}_{\mathrm{AEDT}}$ that these cause are seen in Figure 3, in which the lunch break time of 11.0011.30 shows the maximum difference for all lunch breaks, with less diffuse UV exposure experienced for AEDT. However, when the values of $U_{\mathrm{AEST}}-\mathrm{UV}_{\mathrm{AEDT}}$ are averaged over the five months, the largest influence is the timing of the break with respect to solar noon. Indoor workers who take their breaks outside in a shaded area may have a change in their exposure to diffuse UV due to a shift to daylight saving time, however the magnitude of this change and whether it is a positive or negative change in exposure will depend on the timing of the break. The increase in diffuse UV exposure due to the afternoon break may be negated by the decrease in exposure due to the morning break. In this case, the effect on diffuse UV exposures due to changing to AEDT will be minimal.

Nevertheless, for the states and countries that change to daylight saving time, careful adjustment of the break times spent outdoors by indoor workers has the potential to reduce the exposures to diffuse erythemal UV. A combination of a morning break that is as early as possible, a meal break that is before solar noon and the reduction in the length of the afternoon break that is spent outdoors has the potential to reduce diffuse UV exposures to indoor workers who spend break times in shade outdoors. Conversely, if there is a longer break time in the afternoon than in the morning and the lunch break is taken after solar noon, this has the potential to increase the diffuse 
UV exposures to this group. Further research may also include an investigation into business work practices and consider if the role of daylight saving time is taken into account for staff taking meal break times outside. Depending on UV exposure desired or not desired the meal break times for an individual should be carefully considered.

Acknowledgements: The authors acknowledge the technical support within the Faculty of Sciences, USQ for this project. 


\section{References}

[1] Parisi AV, Kimlin MG. Effect of meal break times on solar UV exposure to school children in a southeast Queensland summer month. Environmetrics 2000; 11(5): 563-570.

[2] Moise AF, Aynsley R. Ambient ultraviolet radiation levels in public shade settings. Int J Biometeorol 1999; 43: 128-138.

[3] Blumthaler M, Ambach W. Spectral measurements of global and diffuse solar ultraviolet-B radiant exposure and ozone variations. Photochem Photobiol 1991; 54(3): 429-432.

[4] Grant RH, Gao W. Diffuse fraction of UV radiation under partly cloudy skies as defined by the Automated Surface Observation System (ASOS). J Geophys Res 2003108(D2), 4046, doi:10.1029/2002JD002201.

[5] Young RW. The family of sunlight-related eye diseases. Optom Vis Sci 1994; 71(2):125-144.

[6] West SK, Duncan DD, Munoz B, Rubin GS, et al. Sunlight exposure and the risk of lens opacities in a population based study. JAMA - Journal of the American Medical Association 1998; 280(8): 714-718.

[7] Holick MF. Sunlight and vitamin D for bone health and prevention of autoimmune diseases, cancers, and cardiovascular disease. Am J Clin Nutr 2004; 80(S): 1678S-1688S.

[8] The Cancer Council Australia. 2007 Available at: http://www.cancer.org.au//File/PolicyPublications/PSRisksBenefitsSunExposur e03May07.pdf (accessed 1 Oct 2007).

[9] Sunsmart, Victoria. 2007 Available at: http://www.sunsmart.com.au/article.asp?ContentID=19april2007 (accessed 1 Oct 2007)

[10] Rock BA. Impact of daylight saving time on residential energy consumption and cost. Energ Build 1997; 25: 63-68.

[11] Coate D, Markowitz S. The effects of daylight and daylight saving time on US pedestrian fatalities and motor vehicle occupant fatalities. Accid Anal Prev 2004; 36: 351-357.

[12] Lahti TA, Leppamaki S, Lonnqvist J, Partonen T. Transition to daylight saving time reduces sleep duration plus sleep efficiency of the deprived sleep. Neurosci Lett 2006; 406: 174-177.

[13] Parisi AV, Kimlin MG, Mainstone JS. Variations in solar erythemal ultraviolet occupational exposure due to daylight saving time in Australia. Rad Prot Australas 1999; 16(3): 13-20. 
[14] CIE (International Commission on Illumination). A reference action spectrum for ultraviolet induced erythema in human skin. CIE J 1987; 6: 17-22.

[15] Turnbull DJ, Parisi AV, Downs NJ. Effect of clouds on the diffuse component of the solar terrestrial UV. Rad Prot Australas 2006; 23: 2-9.

[16] Diffey BL. Stratospheric ozone depletion and the risk of non-melanoma skin cancer in a British population. Phys Med Biol 1992; 37(12): 2267-2279.

[17] Rosenthal FS, West SK, Munoz B, Emmett EA, et al. Ocular and facial skin exposure to ultraviolet radiation in sunlight: a Personal exposure model with application to a worker population. Health Phys 1991; 61: 77-86. 


\section{Figure Captions}

Figure 1 - The diffuse erythemal UV exposures at each five minute period for AEST and AEDT on the cloud free day of 1 February 2004.

Figure 2 - The diffuse erythemal UV exposures at each five minute period for AEST and AEDT on the cloudy day of 27 December 2003.

Figure 3 - The differences of $\mathrm{UV}_{\mathrm{AEST}}$ - $\mathrm{UV}_{\mathrm{AEDT}}$ calculated for the periods of 11.0011.30 AEST, 11.30-12.00 AEST, 12.00-12.30 AEST, 12.30-13.00 AEST, 13.00-13.30 AEST and 13.30-14.00 AEST.

Figure 4 - The averages over each month of $U_{\mathrm{AEST}}-\mathrm{UV}_{\mathrm{AEDT}}$ in the diffuse $\mathrm{UV}$ for four different morning and afternoon tea breaks. The data for the groups 1 to 4 are for the morning break times of 10.00-10.15 AEST, 10.15-10.30 AEST, 10.30-10.45 AEST and 10.45-11.00 AEST respectively. The data for the groups 5 to 8 are for the afternoon break times of 14.30-14.45 AEST, 14.45-15.00 AEST, 15.00-15.15 AEST and 15.15-15.30 AEST respectively.

Figure 5 - The cumulative diffuse $U_{\text {AEST }}-U_{\text {AEDT }}$ exposures for each of the fixed lunch break times.

Figure 6 - The cumulative diffuse $\mathrm{UV}_{\mathrm{AEST}}$ - $\mathrm{UV}_{\mathrm{AEDT}}$ exposures for each of the fixed morning and afternoon tea break times. 


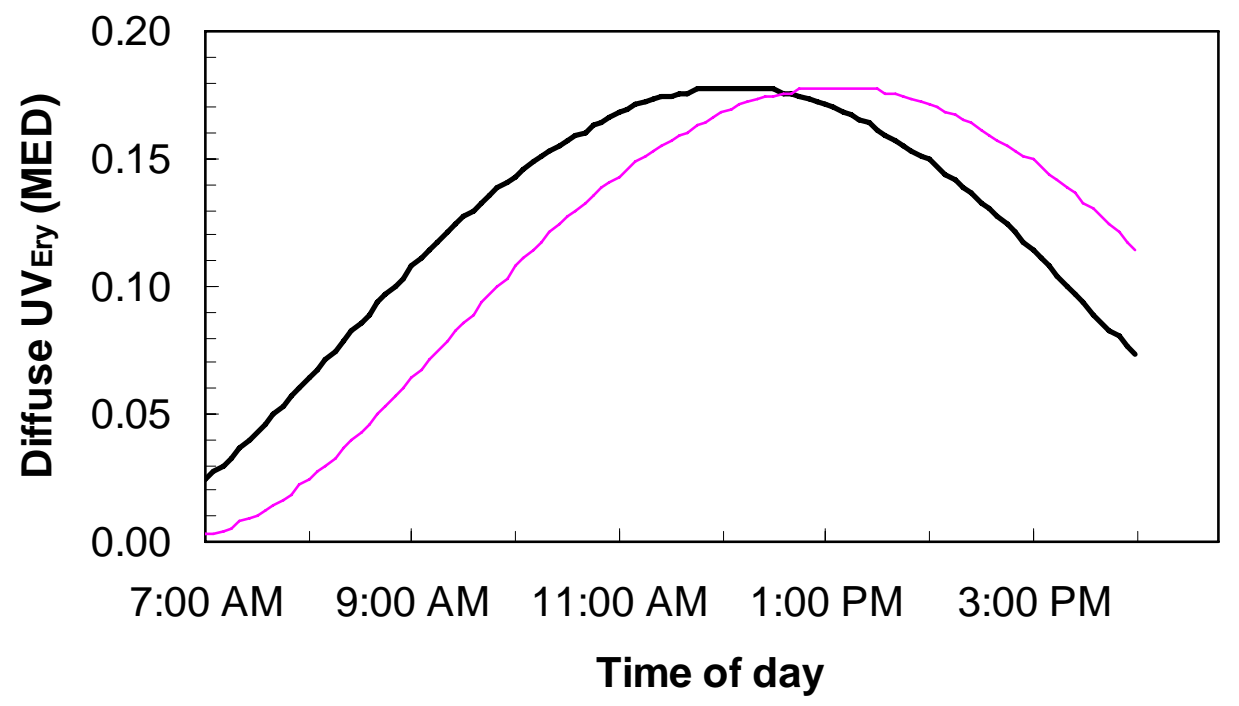

- AEST - AEDT

Figure 1 - The diffuse erythemal UV exposures at each five minute period for AEST and AEDT on the cloud free day of 1 February 2004. 


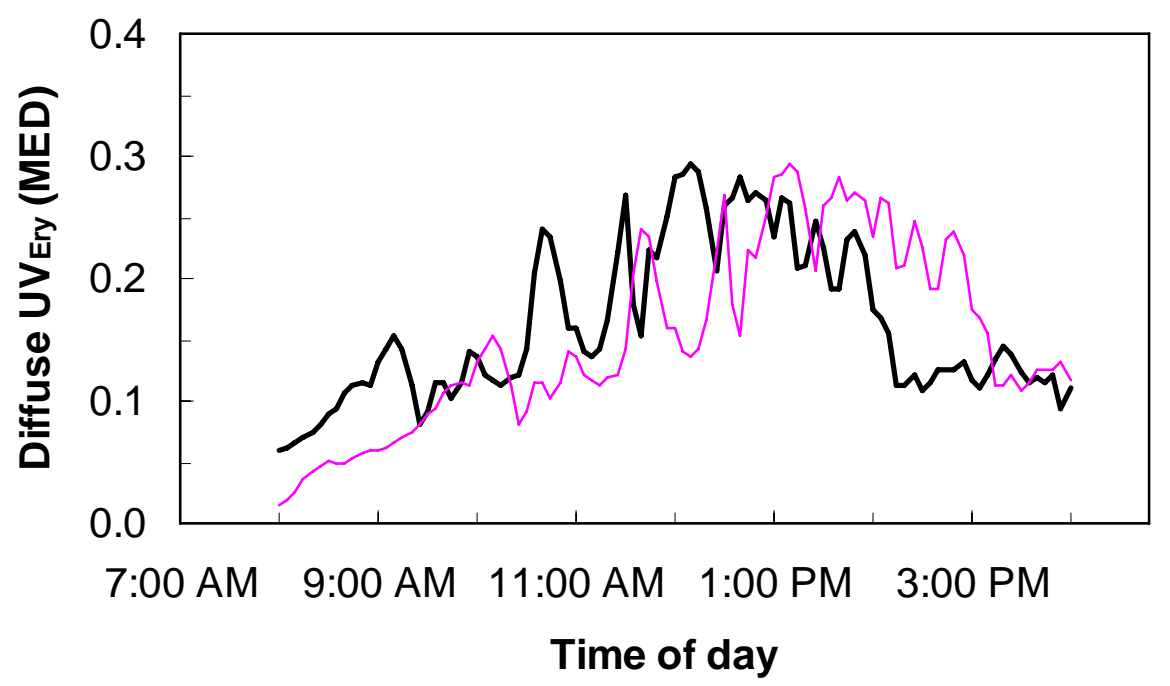

$-\mathrm{AEST} \longrightarrow \mathrm{AEDT}$

Figure 2 - The diffuse erythemal UV exposures at each five minute period for AEST and AEDT on the cloudy day of 27 December 2003. 
(a)

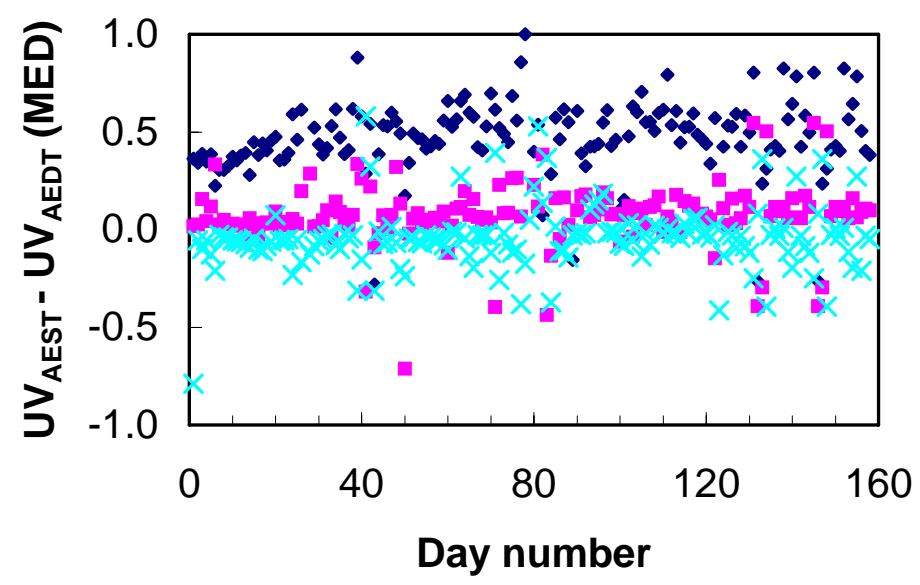

• 11.00-11.30 AEST $\square$ 11.30-12.00 AEST $\times 12.30-13.00$ AEST

(b)

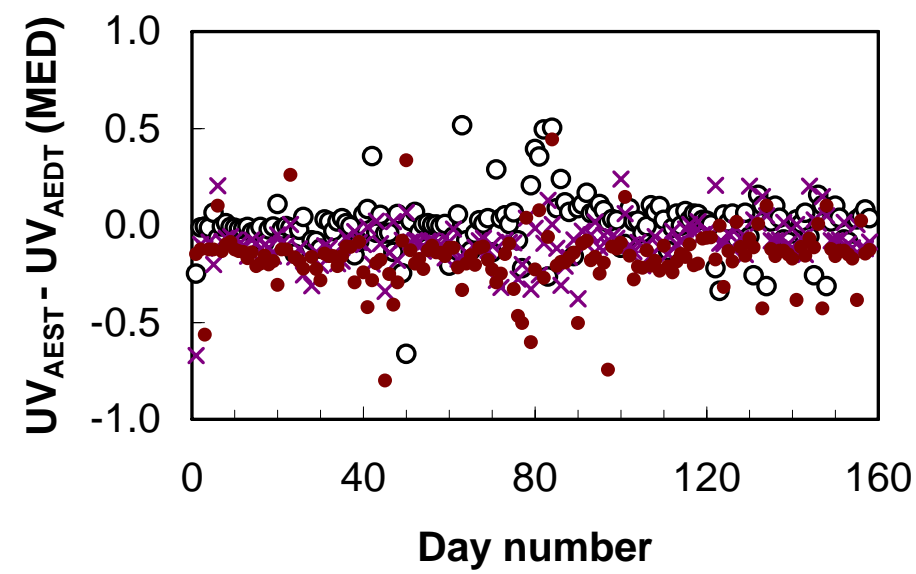

0 12.00-12.30 AEST $\times$ 13.00-13.30 AEST • 13.30-14.00 AEST

Figure 3 - The differences of $\mathrm{UV}_{\mathrm{AEST}}$ - $\mathrm{UV}_{\mathrm{AEDT}}$ calculated for the periods of 11.0011.30 AEST, 11.30-12.00 AEST, 12.00-12.30 AEST, 12.30-13.00 AEST, 13.00-13.30 AEST and 13.30-14.00 AEST. 


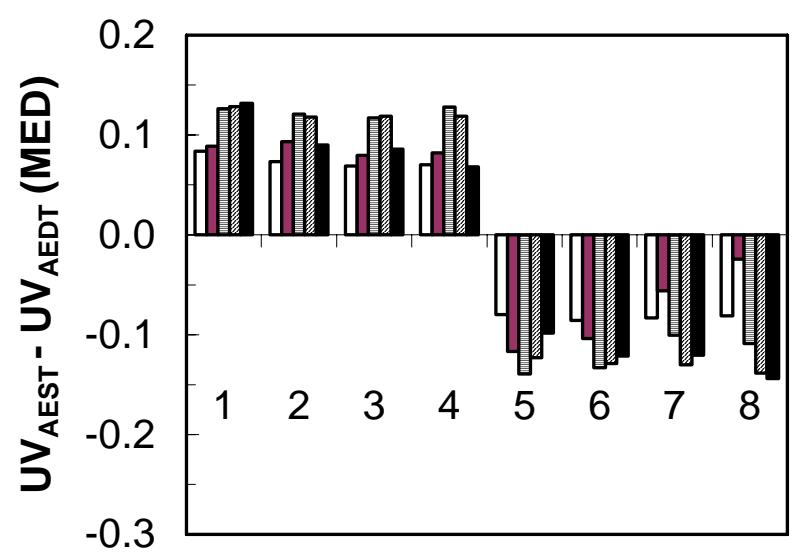

$\square$ Nov $\square$ Dec $\square$ Jan $\square$ Feb $\square$ Mar

Figure 4 - The averages over each month of $U_{\mathrm{AEST}}$ - $\mathrm{UV}_{\mathrm{AEDT}}$ in the diffuse UV for four different morning and afternoon tea breaks. The data for the groups 1 to 4 are for the morning break times of 10.00-10.15 AEST, 10.15-10.30 AEST, 10.30-10.45 AEST and 10.45-11.00 AEST respectively. The data for the groups 5 to 8 are for the afternoon break times of 14.30-14.45 AEST, 14.45-15.00 AEST, 15.00-15.15 AEST and 15.15-15.30 AEST respectively. 


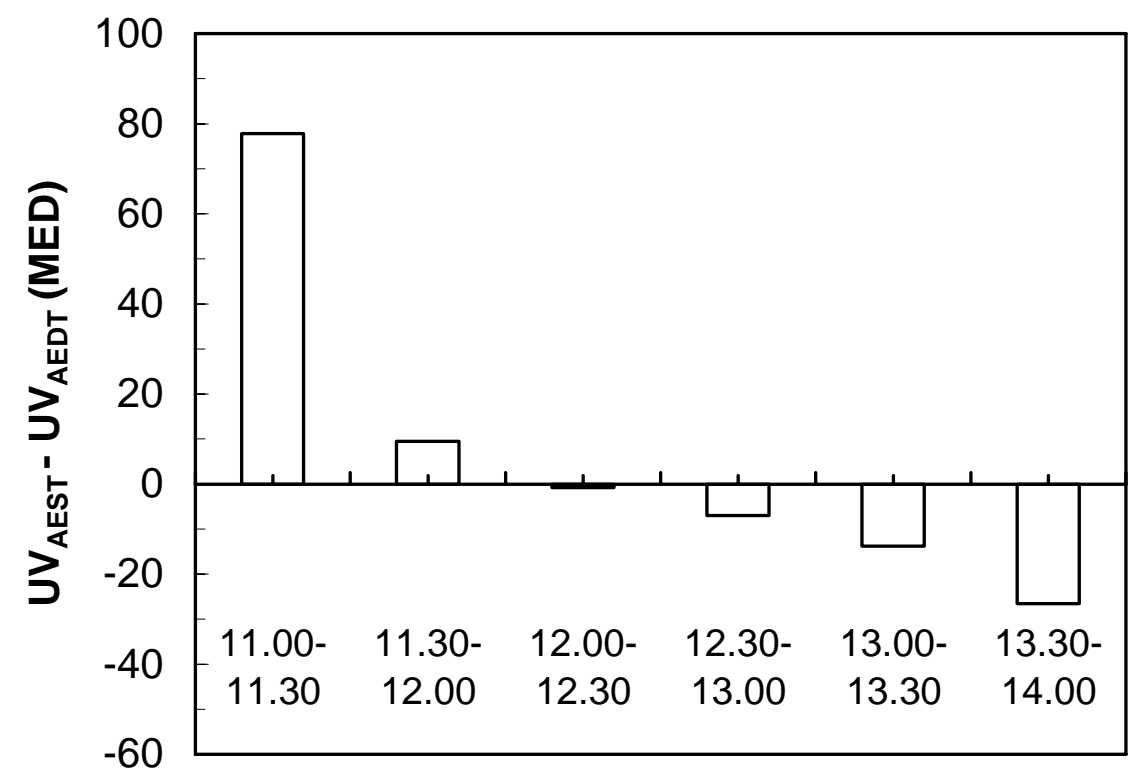

Figure 5 - The cumulative diffuse $\mathrm{UV}_{\mathrm{AEST}}-\mathrm{UV}_{\mathrm{AEDT}}$ exposures for each of the fixed lunch break times. 


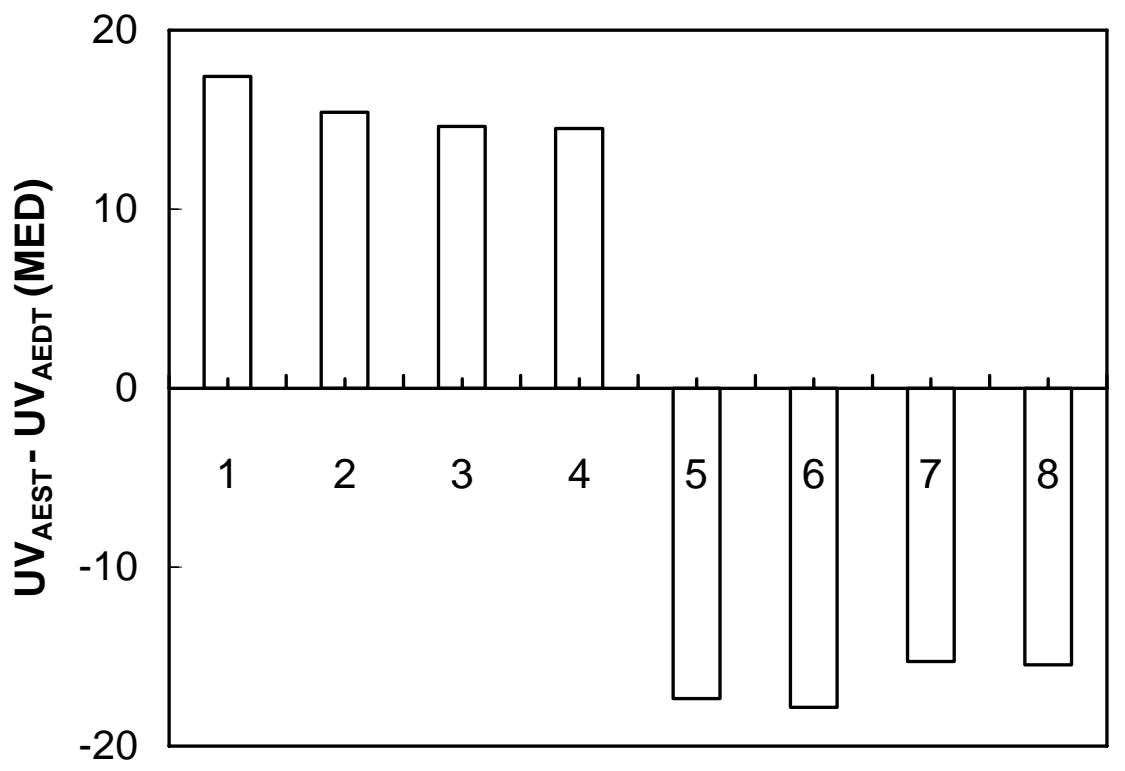

Figure 6 - The cumulative diffuse $\mathrm{UV}_{\mathrm{AEST}}$ - $\mathrm{UV}_{\mathrm{AEDT}}$ exposures for each of the fixed morning and afternoon tea break times. 